\title{
Hochschulszene Sensorik und Mikrosystemtechnik: Wer macht was wie?
}

\author{
Prof. Dr. Andreas Schütze \\ Lehrstuhl für Messtechnik, Fachrichtung Mechatronik, Universität des Saarlandes \\ Universität Campus, Postfach 1511 50, 66041 Saarbrücken
}

\section{Einleitung}

Die Hochschullandschaft in Deutschland ist vielfältig und entwickelt sich, insbesondere durch die umfangreichen Novellierungen und Reformen der letzten Jahre, stürmisch weiter. Neben dem viel diskutierten Bologna-Prozess, verbunden mit der Umstellung der Abschlüsse an Hochschulen auf das angelsächsische System der gestuften Bachelor-/Master-Abschlüsse, der in einem gesonderten Beitrag behandelt wird [1], entwickelt sich die Hochschullandschaft auch durch Schaffung neuer Studienangebote laufend weiter. Der Beitrag soll einerseits einen Überblick geben über Studiengänge, die spezifisch die Mikrosystemtechnik und damit auch besonders die Sensorik adressieren, andererseits typische Modelle vorstellen, über die Unternehmen und Hochschulen in der Ingenieurausbildung zusammenarbeiten. Stichworte, unter der diese Zusammenarbeit Industrie-Hochschule erfolgt, sind vor allem kooperatives bzw. duales Studium. Der Beitrag stützt sich u.a. auf eine Online-Befragung der VDI/VDE-IT sowie eigene Recherchen, erhebt dabei aber keinen Anspruch auf Vollständigkeit, da hierfür die Landschaft insgesamt zu vielfältig ist.

\section{Studiengänge Sensorik und Mikrosystemtechnik im deutschsprachigen Raum}

Bereits seit vielen Jahren werden in Deutschland Studiengänge mit den Schwerpunkten Mikrosystemtechnik und Sensorik angeboten und zwar sowohl an Universitäten als auch Fachhochschulen. Getrieben wurde diese Entwicklung u.a. durch die begleitenden Maßnahmen des BMBF zu den Förderprogrammen Mikrosystemtechnik, da von Anfang an die Erkenntnis zugrunde lag, dass die Umsetzung von Forschungsergebnissen in Produkte und damit letztlich in Gewinne nur gelingen kann, wenn ausreichend Fachkräfte für die industrielle Umsetzung zur Verfügung stehen. Gerade die Hochtechnologiebereiche haben in den vergangenen Jahren schmerzhaft erfahren müssen, wie schwierig die Gewinnung von qualifizierten Fachkräften auf allen Ebenen des Ausbildungssystems ist und wie sehr dieser Fachkräftemangel Innovationen verzögert oder behindert. Bereits frühzeitig entstand dabei eine Landkarte der Studienangebote im Bereich Mikrosystemtechnik durch die VDI/VDE-IT, siehe Abb. 1. In der Folge wurden in den Jahren 2003 bis 2008 die Aus- und Weiterbildungsnetzwerke für die Mikrosystemtechnik (AWNET) gefördert, die sich den großen Themen Nachwuchssicherung, Fort- und Weiterbildung, Lehrund Lernmaterialien sowie Vernetzung von Akteuren regional und überregional verschrieben haben [2, 3]. Dabei wurde das gesamte Spektrum der Ausbildungswege vom Mikrotechniker/Mikrotechnologen bis zur Promotion betrachtet, ebenso wie die Übergangs- und Aufstiegsmöglichkeiten, Abb. 2.

In einer Online-Befragung und -Recherche, durchgeführt durch die VDI/VDE-IT im Jahr 2008, wurde das Gesamtbild aktualisiert bzw. wurden die Hochschulen nach der Relevanz verschiedener Aspekte befragt. Neben der weiter wachsenden Zahl relevanter Studienangebote im Bereich Mikrosystemtechnik, siehe Abb. 3, wurde die bereits weit fortgeschrittene Umstellung der Studiengänge auf das gestufte BachelorMaster-System festgestellt (57\% der Studienangebote sind bereits vollständig umgestellt, weitere $21 \%$ zum überwiegenden Teil). Dennoch stellt auch heute noch die Umstellung ein wichtiges Diskussionsthema im Hochschulkreis dar, insbesondere weil es keine einheitlichen Vorgaben zu Struktur und Inhalten der Studiengänge gibt und noch keine gemeinsamen Qualitätsmaßstäbe etabliert wurden. 


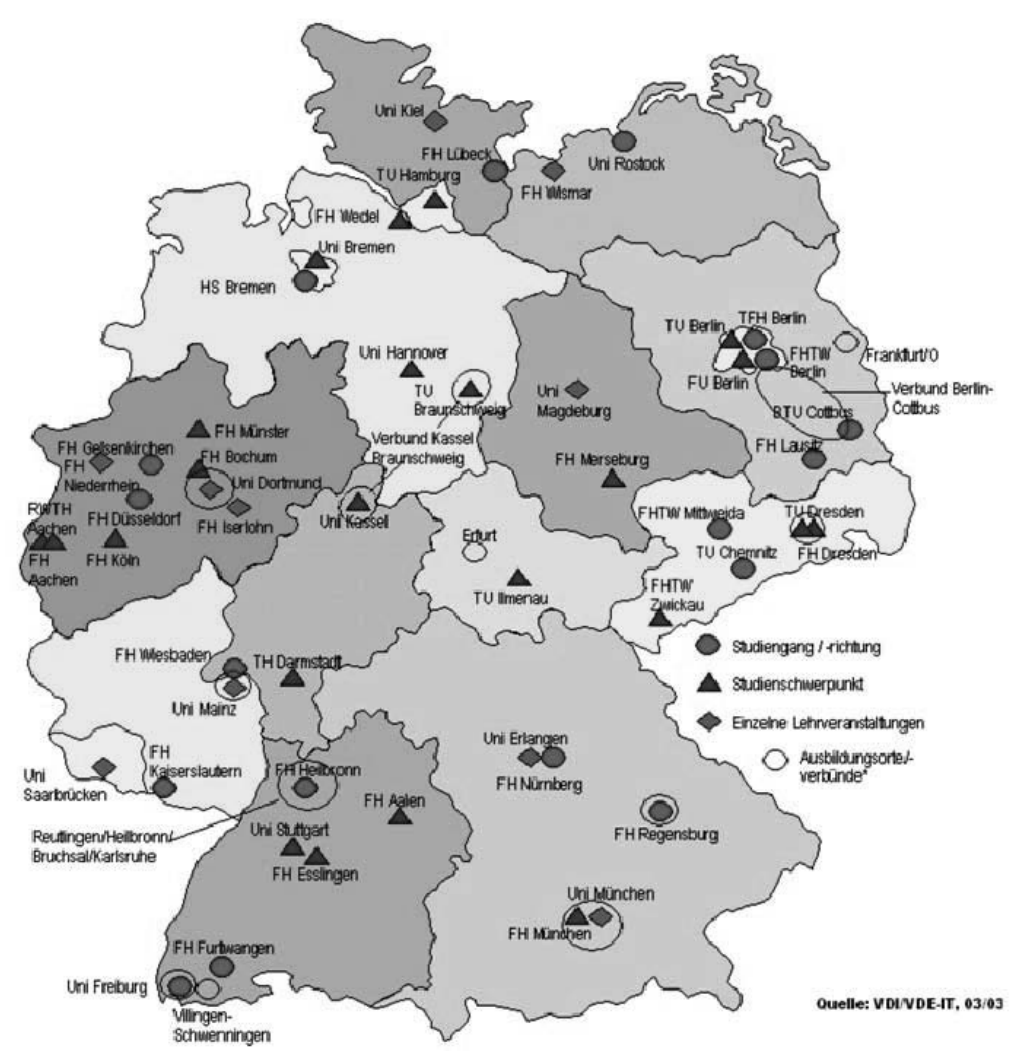

Abb. 1: Landkarte der Mikrosystemtechnik-Studienangebote in Deutschland. Neben dedizierten Studiengängen gab und gibt es eine Vielzahl von MST-Schwerpunkten bzw. einzelnen MST-Lehrveranstaltungen, Stand 2003.

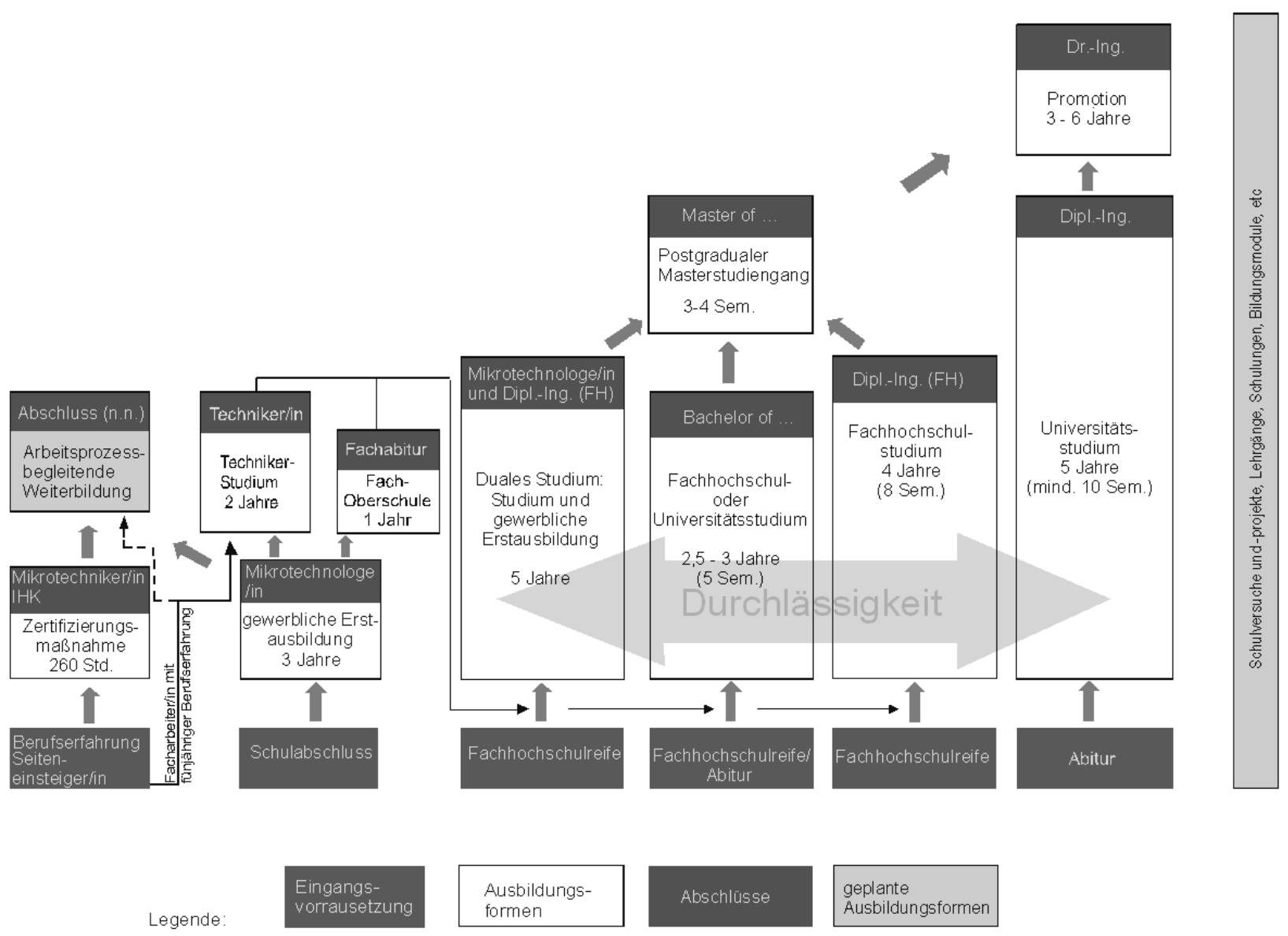

Abb. 2: Übersicht über Ausbildungsgänge im Bereich Technik allgemein und Sensorik/Mikrosystemtechnik im Besonderen mit Übergangs- und Aufstiegsmöglichkeiten. Entnommen aus [3] 


\section{Studiengänge Mikrosystemtechnik ( $n=113)$}

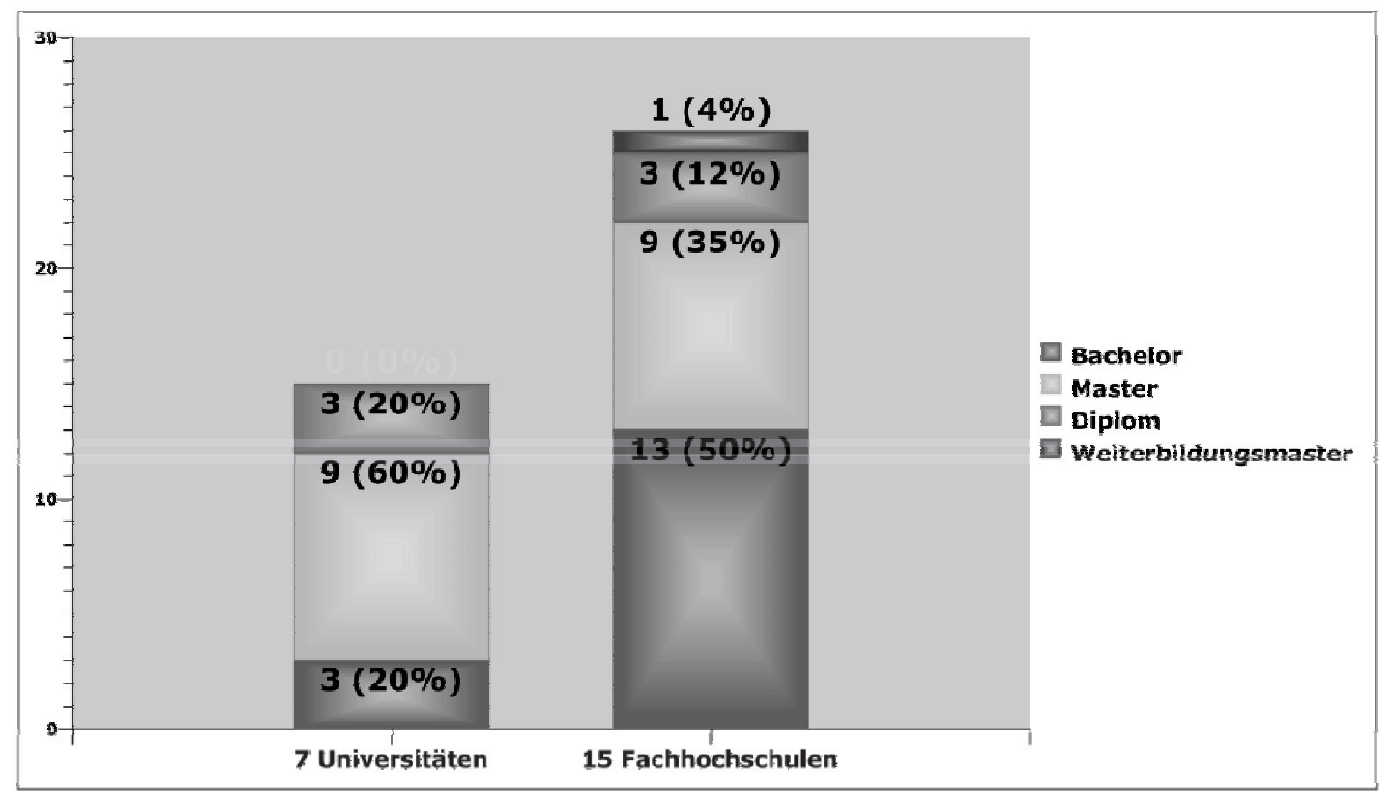

Abb. 3: Aktueller Stand der MST-Studiengänge an Universitäten und Fachhochschulen in Deutschland, aufgeschlüsselt nach Abschlüssen. Insgesamt werden an 113 (von insgesamt 266 Hochschulen mit natur- und/oder ingenieurwissenschaftlichen Studienangeboten) MST-relevante Inhalte vermittelt. Neben den oben aufgeführten 41 MST-Studiengängen an 22 Hochschulen gibt es weitere 165 Studiengänge mit MST-Spezialisierung/ Vertiefung/Schwerpunkt an 76 Hochschulen.

Als besonders bedeutsam werden im Umfeld der Hochschulen heute die Themen Nachwuchssicherung (nicht weniger als 95\% aller Hochschulen schätzen dies als relevant oder sehr relevant ein) und, damit einhergehend, die Reduzierung der Studienabbrecherquoten sowie die Kooperationen im Bereich Lehre angesehen. Zwei Drittel aller Hochschulen kooperieren heute bereits im Rahmen der MST-Ausbildung mit anderen Hochschulen und zwar zum Großteil international. Als wesentliche Vorteile der Kooperationen werden dabei genannt die größere Attraktivität für Studierende (letztlich wieder aufgrund des allgemeinen Nachwuchsmangels) sowie der Zugang zu Infrastruktur (insbesondere Reinräumen), die an der eigenen Hochschule nicht vorhanden ist. Ein erfolgreiches Modell stellt hier beispielsweise die Aus- und Weiterbildungsfoundry pro-mst an der FH Kaiserslautern, Standort Zweibrücken dar [4, 5]. Bemerkenswerterweise wird der Aspekt des Zugangs zu Infrastruktur durch Kooperationen sogar von den Hochschulen als noch relevanter angesehen, die heute noch nicht kooperieren, so dass hier in der Zukunft weitere Aktivitäten sinnvoll und notwendig sind. Gerade für die MST stellt der Zugang zu modernster Technologie einen wichtigen Aspekt der Qualitätssicherung auch in der Lehre dar.

\section{Kooperationsmodelle zwischen Industrie und Hochschulen in Sensorik und MST}

An vielen Hochschulen gibt es heute eine mehr oder weniger intensive Verflechtung der akademischen Ausbildung in den Ingenieurwissenschaften mit industriellen Partnern. Hintergrund ist zum einen das große Interesse der Industrie, die passenden Absolventen für ihre Tätigkeit direkt von den Hochschulen zu erhalten, ohne diese intensiv nachschulen zu müssen. Daneben spielen aber auch andere Aspekte eine Rolle, z.B. das Interesse der Studierenden nach verbesserten Chancen auf dem Arbeitsmarkt oder die rasche technologische Entwicklung, die es den Hochschulen nicht mehr erlaubt, in der Breite die neueste technologische Ausstattung für die Ausbildung vorzuhalten. Hier bietet die Kooperation mit Firmen oder anderen Ausbildungseinrichtungen die Möglichkeit, aktuelles High-Tech-Equipment in die 
Ausbildung der Studierenden einzubeziehen, und zwar nicht nur in (Forschungs-)Bereichen, in denen die Hochschule selbst stark ist, sondern in deutlich größerer Breite.

Versucht man eine (notgedrungen grobe) Klassifizierung der Kooperationen zwischen Hochschulen und Industrie, dann zeigt sich ein breites Spektrum, das auch von der Zielsetzung der Ausbildung stark geprägt ist. Während an den vornehmlich anwendungsorientiert ausgerichteten Fachhochschulen eine enge Verknüpfung - meist mit ausgeprägtem lokalen Schwerpunkt - bereits traditionell dadurch gegeben ist, dass der überwiegende Anteil der Abschlussarbeiten direkt in der Industrie angefertigt wird, fokussiert sich die Kooperation der forschungsorientierten Universitäten mit Unternehmen auf gemeinsame F\&EProjekte, durch die insbesondere neue Technologien in die Unternehmen Eingang finden. Neben diesen meist bilateralen Kooperationen zwischen einzelnen Arbeitsgruppen und Unternehmen gibt es stärker formalisierte und inhaltlich umfangreichere Zusammenarbeit zwischen Studiengängen bzw. den sie tragenden Fakultäten/Fachbereichen und Unternehmen, die im Idealfall das ganze Studium umfassen bzw. begleiten. Zu nennen sind hier insbesondere folgende Modelle:

- Kooperatives Studium

Typischerweise (es gibt eine Vielzahl von hochschulspezifischen Lösungen) erfolgt die Kooperation im Rahmen eines trilateralen Vertrags zwischen Studierenden, Firma und Hochschule. Der/die Studierende absolviert sein Studium nach denselben Regeln und im selben Zeitrahmen wie normal eingeschriebene Kommilitonen, erhält allerdings von der Firma einen Vertrag mit einer Vergütung, die die Lebenshaltungskosten während des Studiums weitgehend deckt. Meist orientiert sich die Höhe der Vergütung an der Ausbildungsvergütung, wird aber nicht im Rahmen eines Arbeitsvertrags gewährt, sondern als Stipendium bzw. Aufwandsentschädigung. Im Gegenzug verpflichtet sich der Studierende, die im Studium abzuleistenden Praktika und Arbeiten (Studien- und Diplomarbeit bzw. Bachelor- und Masterarbeit) im bzw. für das Unternehmen zu absolvieren sowie zusätzlich in einem gewissen Umfang (in der Regel ein Tag pro Woche) im Unternehmen tätig zu sein. Im Idealfall durchläuft der Studierende damit parallel zu seinem Studium verschiedene Abteilungen im Unternehmen und lernt so das gesamte Spektrum und Arbeitsgebiet kennen. Nach Abschluss des Studiums ist damit für den Absolventen und die Firma gut planbar, in welchem Bereich ein Einsatz sinnvoll und beiderseits wünschenswert ist. Die Firma erhält im Rahmen des Vertrages ein gewisses „Vorkaufsrecht", d.h. wenn die Firma nach Abschluss des Studiums einen Arbeitsplatz anbietet, muss der Absolvent diesen annehmen oder einen Teil der erhaltenen Gelder zurückzahlen.

- Duales Studium

Das duale Studium geht noch einen Schritt weiter und verbindet das Hochschulstudium mit einer gewerblichen Ausbildung zum Facharbeiter, z.B. in einem metallverarbeitenden oder elektrotechnischen Beruf. Dafür schließen die Studierenden für die gesamte Laufzeit des Studiums einen Ausbildungsvertrag mit einem ausgewählten Unternehmen ab. Der Facharbeiterabschluss und der Hochschulabschluss werden sukzessive erreicht, siehe Abb. 4 [6]. Durch die Integration ergibt sich eine Ausbildungszeitverkürzung gegenüber dem vielfach praktizierten Modell des Studiums nach Abschluss einer Ausbildung.

Noch einen Schritt weiter geht das Modell StudiumPlus getragen vom Wissenschaftlichen Zentrum Dualer Hochschulstudien (ZDH) [7]. In einer Kooperation der FH Gießen-Friedberg mit den Industrie- und Handelskammern sowie Unternehmen der Region wurde am Standort Wetzlar eine eigene Ausbildungsstätte für duale Studiengänge verschiedenster, vor allem wirtschaftsnaher Fachrichtungen etabliert. Ein Angebot ist dort der Studiengang Ingenieurwesen/MST mit den Richtungen Mikrosystemtechnik/Optronik, Elektrotechnik sowie Maschinenbau und dem Abschluss Bachelor of Engineering (B.Eng.). 


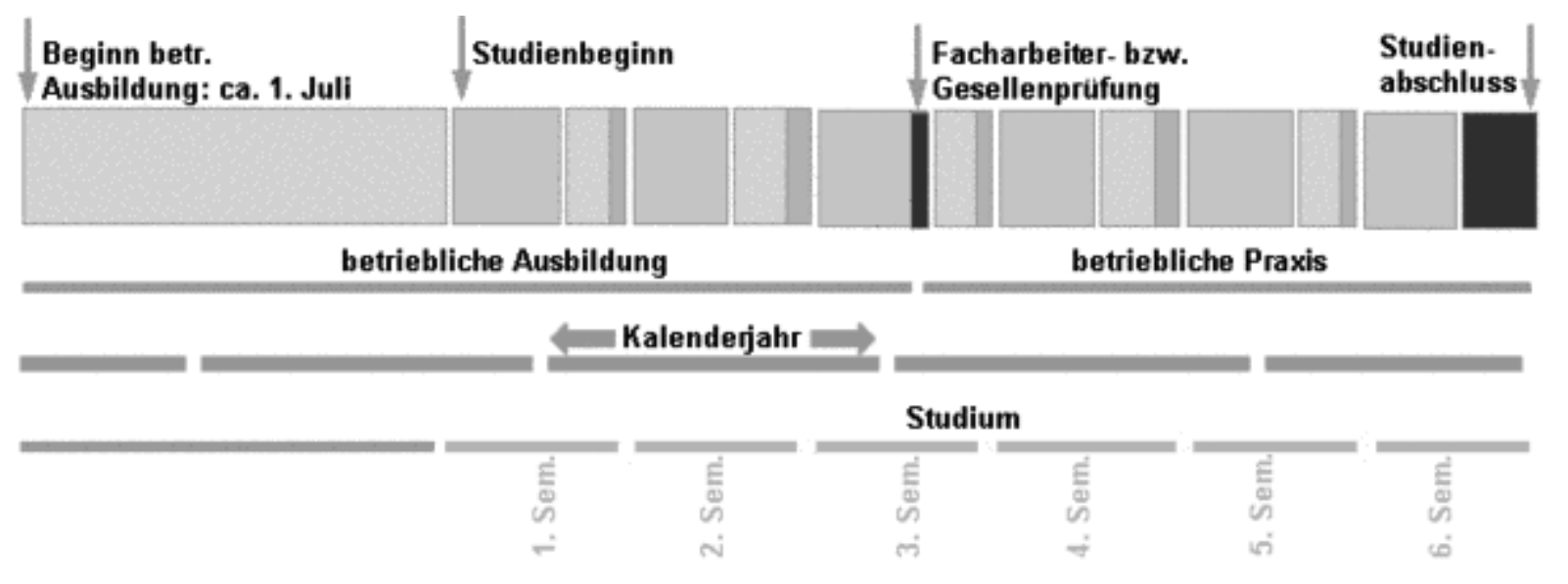

betriebliche Ausbildungszeit

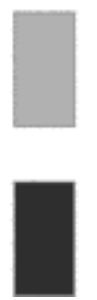

Urlaub

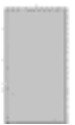

Studium

betriebliche Abschlussp rüfung / Bachelorarbeit

Abb. 3: Typisches Modell für ein duales Studium, das eine berufliche Ausbildung mit einem (Bachelor-)Studium verknüpft [4]. Die Gesamtdauer für beides Ausbildungen beträgt etwa 4 1/2 Jahre und verkürzt damit die Zeit für gegenüber einem Studium nach der Ausbildung um mindestens ein Jahr.

Das kooperative bzw. duale Studium nach diesen bzw. ähnlichen Modellen gibt es an einer ganzen Reihe von Hochschulen, allerdings vornehmlich bzw. im Falle des dualen Studiums sogar ausschließlich an Fachhochschulen. Dies ist bedingt durch die große Anwendungsspezifität, die beim kooperativen Studium erwünscht, beim dualen Studium mit Verknüpfung von gewerblicher und akademischer Ausbildung zwingend ist. Ein kooperatives Studium gibt es aber beispielsweise auch an der Universität des Saarlandes in Verbindung mit ausgewählten Firmen in den Ingenieurwissenschaften und der Informatik. Es besteht hier aber Konsens zwischen Unternehmen und Universität, dass das Durchlaufen der unterschiedlichen Abteilungen im Unternehmen praktisch einem vorweggenommenem Trainee-Programm gleichkommen soll und dass Diplom- und Masterarbeiten dem üblichen hohen wissenschaftlichen Anspruch genügen müssen. Die Abschlussarbeiten werden daher in der Regel nicht im Unternehmen angefertigt, sondern in einer universitären Arbeitsgruppe, beschäftigen sich aber mit einer gemeinsam mit dem Unternehmen definierten Fragestellung und zielen auf einen direkten Transfer von Know-how und Technologie ins Unternehmen. Eine zweite Besonderheit der Vereinbarung besteht darin, dass das „Vorkaufsrecht" der Firma im Hinblick auf eine mögliche Promotion nach Abschluss des Studiums eingeschränkt ist: strebt der Absolvent eine Promotion an, müsste die Firma den Abschluss der Promotion abwarten, bevor sie den Absolventen einstellen kann.

\section{Fazit und Ausblick}

Gerade die derzeit laufenden Reformbemühungen an den Hochschulen - auch aber nicht ausschließlich im Rahmen des Bologna-Prozesses [1] - führen zu einer laufenden Veränderung der Studienangebote. Dabei spielt auch der Ingenieurmangel eine große Rolle: vielfach beobachtet man einen regen Zuspruch der Studienanfänger bei neuen Studiengängen insbesondere mit „moderneren“ Bezeichnungen gegenüber den klassischen Studiengängen Elektrotechnik/Informationstechnik und Maschinenbau/Verfahrenstechnik. Dies übt gleichzeitig Druck auf die (auch) an Studienanfängerzahlen gemessenen Fakultäten/ 
Fachbereiche aus, neue Studienangebote zu entwickeln, um im Wettbewerb der Hochschulen bestehen zu können. Dabei gilt es allerdings abzuwägen zwischen dem insbesondere seitens der Universitäten verfolgten Anspruch einer breiten, theoretisch fundierten Ausbildung, die eben nicht auf ein schmales Betätigungsfeld der Absolventen abzielt, und dem Wunsch seitens der Studierenden, aber auch seitens der Industrie nach einer spezifischeren bzw. passgenaueren Ausbildung. In diesem Spannungsfeld werden sich Studiengänge wie Mikrosystemtechnik und beispielsweise auch Mechatronik, die in ähnlicher Weise Themen der beiden klassischen Ingenieurdisziplinen verbinden, sicher dauerhaft etablieren. Bei anderen Studiengängen besteht die Gefahr, dass diese schnell wieder verschwinden, wenn die Themen nicht mehr en vogue sind und damit die Studienanfängerzahlen wieder sinken bzw. sich herausstellt, dass die Industrie mit den Absolventen nicht unmittelbar etwas anfangen kann, weil z.B. die Studieninhalte nicht bekannt sind. Aus diesem Grund ist es wünschenswert bei der Reform bestehender bzw. Etablierung neuer Studiengänge die Industrie grundsätzlich in die Gestaltung einzubinden, allerdings nur, wenn seitens der Industrie auch die langfristige Perspektive der Hochschulen angemessen berücksichtigt wird. Hier sind neben großen Firmen und Verbänden gerade auch kleine und mittlere Unternehmen als Diskussionspartner gefragt, da deren Sicht sich doch erheblich von Großunternehmen unterscheidet.

Auch bei den weitergehenden Kooperationsmöglichkeiten sind KMU gefragt, wobei diese sich meist in größeren Gruppen zusammenschließen müssen, damit eine ausreichende Kontinuität gesichert ist. Ein Beispiel hierfür ist sicher das StudiumPlus in Wetzlar, das maßgeblich von regionalen Mittelständlern getragen wird. Trotz des deutlichen Übergewichts der Angebote bei Fachhochschulen sei aber ausdrücklich betont, dass auch eine Kooperation zwischen Universitäten und KMU durchaus sinnvoll und für beide Seiten befruchtend sein kann, wenn ebenfalls beidseits auf einen angemessenen Interessenausgleich geschaut wird. Für Unternehmen heißt das insbesondere, dass von Universitäten keine ausschließlich oder primär auf die Anwendung fokussierte Ausbildung erwartet werden darf, da hier die Forschungsorientierung klar im Vordergrund steht. Dies bietet aber auch und gerade KMU Zugang zu neuesten Technologien und innovativen Köpfen. Im Gegenzug sollten sich Universitäten aber auch öffnen für die Interessen und Fragestellungen von KMU und dürfen diese nicht von oben herab behandeln. Natürlich kann ein kleines Unternehmen nicht in großem Umfang Forschungsaufträge vergeben, aber z.B. bei der Nachwuchswerbung kann die Kooperation mit einem lokalen Mittelständler, Stichwort kooperatives Studium, sehr hilfreich sein. Da Studieninteressenten großes Interesse an diesen Angeboten zeigen, droht sonst eine verkehrte Welt: aufgrund großer Nachfrage können Firmen sehr hohe Anforderungen an Studieninteressenten im kooperativen Studium stellen, die abgelehnten Bewerber streben dann in die nicht zulassungsbeschränkten universitären Studiengänge.

\section{Literatur}

[1] Gerald Gerlach und Michael Schanz: Bolognaprozess und Bachelor-/Masterabschlüsse: Worauf müssen sich Unternehmen einstellen?, SENSOR+TEST 2009, Sondersession Aus- und Weiterbildung, Nürnberg, 26.28.05.2009.

[2] http://www.mst-ausbildung.de

[3] AWNET-Zwischenbilanz 2003-2005, AWNET-Geschäftsstelle (Hrsg.), 2005, ISBN 3-89750-143-0.

[4] http://www.pro-mst.de/

[5] A. Picard et al.: Die Aus- und Weiterbildungsfoundry pro-mst, Beitrag zum Workshop Hochschulausbildung, in: Nicolas Hübener, Andreas Schütze (Hrsg.), Aus- und Weiterbildung in Hochtechnologiefeldern Fachkräftesicherung in Neuen Technologien, Dokumentation zum gleichnamigen Kongress, Berlin, 29.30.11.2007, AWNET, Berlin, ISBN 978-3-89750-149-2, S. 70 - 73.

[6] http://www.hs-owl.de/studium/studienangebot/koop-studium/zwei-modelle.html

[7] http://www.studiumplus.de 